\title{
What Is Lost in Postgraduate Residency During COVID-19 Times?-a Resident Perspective on Diagnostic Error
}

\author{
M. Shuriya Prabha ${ }^{1}$ V. Dinesh Kumar ${ }^{2}$ (I) \\ Published online: 24 August 2020 \\ (C) International Association of Medical Science Educators 2020
}

\section{Dear editor,}

We read the student's perspective of learning amidst crisis depicting the hiccups endured to the clinical clerkships at recent times and wish to document resident's viewpoint on the ongoing scenario of educational disruption [1]. In addition to the uncertainty regarding the time of resumption of routine clinics, tremendous loss in psychomotor skills training, and fatigable hours of posting in critical units, the cardinal loss incurred is the blunt of clinical reasoning skills, which forms the crux of evidence-based medicine. Apart from the internal determinants such as motivation, cognitive quest, and burnout, the process of clinical reasoning can also be influenced by external influencers such as time constraints, environmental cues, and lack of investigations [2]. The pandemic outbreak has posed as an additional extrinsic constraint to the process of diagnostic reasoning owing to the fact that physical examination is done with 'social distancing' protocols with more emphasis being levied upon 'unwarranted' investigations.

In most healthcare organization, patients are classified into COVID-19 suspects and non-COVID-19 suspects upon arriv$\mathrm{al}$ at the emergency/outpatient wing itself and this stringent bifurcation narrows down the further ramification of diagnostic reasoning made by a resident. If a patient is hailing from an area where few cases infected with virus had been residing, we either jump into assumptions before developing the complete illness script or search for the whole panel of atypical presentations of contagion described in the literature for completing the process of 'dichotomization'. In few worse case scenarios, even if the virus is proven to be negative, patients with one or other trivial respiratory symptoms are subjected to computed

\section{Dinesh Kumar}

dinesh.88560@gmail.com

1 Rajah Muthiah Medical College, Chidambaram, Tamil Nadu, India

2 Jawaharlal Institute of Postgraduate Medical Education and Research, Puducherry, India tomography scans to clear the air of suspicion. To be precise, the resident's reasoning process has got considerably fogged by the subconscious and tepid bias invoked by the pandemic.

While being in paediatric casualty duty, we encountered a 6-year-old boy presenting with symptomatology of breathlessness. The workup which had routinely commenced got halted by the information that he was residing in COVID-19 high prevalence zone, i.e. an area where few active cases has been recently detected. Though the chest radiograph showed evident pleural effusion and despite not having typical signs such as fever, myalgia, and loss of smell, we had to pause the workup and refer him to a COVID-19 designated unit in order to rule out the possibility of infection. Owing to this, the pleural tapping was unduly delayed because performing invasive procedures in patients with virus positivity mandated a different PPE protocol and should not be done at routine casualty service. After hours of delay associated with confirming the boy to be virus-negative, the pleural tap was performed and the laboratory picture was suggestive of tuberculosisassociated pleural effusion. Upon further probing, we obtained the history of his father suffering from tuberculosis from whom the index case would have likely contracted the disease. We got completely annoyed for not being able to reason out in conventional manner which we used to do and uncalculatingly missing the vital cue.

The abovementioned case is a characteristic exemplar of availability bias whereby the presumption is prematurely arrived based on how easily they come in mind because of the confounding influence of the other cases or scenarios which have been recently seen [3]. In the diagnostic process, we tend to match the presenting clinical features to the illness scripts which we have developed within ourselves over a period of time. The situated cognition of pandemic 'intuitively' pushes us to fit the mimicking clinical features, and this subconscious blinding leads to diagnostic oversight of endemically prevalent respiratory conditions [4]. Similarly, blind anchoring to the pandemic outbreak protocol might prevent from exploring other genuine alternatives and escape from sense of 
uncertainty [5]. The chronology of gathering cues also matters in reducing diagnostic error, and in our case, the history gathered initially was more suggestive of confirming a hypothesis, i.e. COVID-19 positivity rather than refuting it amounting to confirmation bias [6].

To conclude, the art of diagnostic reasoning with utmost precision is the most valuable thing which a resident could learn and this could always be deceived by undercurrent cognitive biases. Continuous downpour of pandemic-related information and protocol mandates has blurred our mindful intellect to focus upon other associated information and therefore leading to a diagnostic error. We, therefore, wish to document that learning amidst crisis also includes learning from our own errors and is a sharp learning experience for not falling prey to the obtrusive biases in the wave of uncertainty. In an optimistic note, we might never encounter such uncertain rounds anytime in the future for learning clinical equipoise greater than ever.

\section{Compliance with Ethical Standards}

Conflict of Interest The authors declare that there is no conflict of interest.

Ethical Approval and Informed Consent This submission is a letter to the editor, and therefore, ethical approval is not required. Though the anecdote mentioned over here never reveals any information pertaining to identity of the case, we had obtained verbal informed consent following prevailing ethical guidelines from the parent for quoting in our letter.

\section{References}

1. Alexander SM. Learning amidst a crisis - a student perspective on COVID-19. Med Sci Educ. 2020. https://doi.org/10.1007/s40670020-01037-5.

2. Young M, Thomas A, Gordon D, Gruppen L, Lubarsky S, Rencic J, et al. The terminology of clinical reasoning in health professions education: implications and considerations. Med Teach. 2019;41(11):1277-84.

3. Croskerry P. The importance of cognitive errors in diagnosis and strategies to minimize them. Acad Med. 2003;78:775-80.

4. Coleman JJ, Manavi K, Marson EJ, Botkai AH, Sapey E. COVID19: to be or not to be; that is the diagnostic question. Postgrad Med J. 2020;96(1137):392-8. https://doi.org/10.1136/postgradmedj-2020137979.

5. Zagury-Orly I, Schwartzstein RM. Covid-19 - a reminder to reason. N Engl J Med. 2020;383(3):e12. https://doi.org/10.1056/ NEJMp2009405.

6. Nickerson RS. Confirmation bias: a ubiquitous phenomenon in many guises. Rev Gen Psychol. 1998;2:175-220.

Publisher's Note Springer Nature remains neutral with regard to jurisdictional claims in published maps and institutional affiliations. 